\title{
Charged Relativistic Fluids and Non-linear Electrodynamics
}

\author{
T. Dereli* \\ Department of Physics, Koç University, 34450 Istanbul, Turkey \\ R. W. Tucker ${ }^{\dagger}$ \\ The Cockcroft Institute, Daresbury, UK \\ and \\ Department of Physics, Lancaster University, Lancaster, UK
}

\begin{abstract}
The electromagnetic fields in Maxwell's theory satisfy linear equations in the classical vacuum. This is modified in classical non-linear electrodynamic theories. To date there has been little experimental evidence that any of these modified theories are tenable. However with the advent of high-intensity lasers and powerful laboratory magnetic fields this situation may be changing. We argue that an approach involving the self-consistent relativistic motion of a smooth fluid-like distribution of matter (composed of a large number of charged or neutral particles) in an electromagnetic field offers a viable theoretical framework in which to explore the experimental consequences of non-linear electrodynamics. We construct such a model based on the theory of Born and Infeld and suggest that a simple laboratory experiment involving the propagation of light in a static magnetic field could be used to place bounds on the fundamental coupling in that theory. Such a framework has many applications including a new description of the motion of particles in modern accelerators and plasmas as well
\end{abstract}

*tdereli@ku.edu.tr

${ }^{\dagger}$ r.tucker@lancaster.ac.uk 
as phenomena in astrophysical contexts such as in the environment of magnetars, quasars and gamma-ray bursts.

Classification Numbers: 02.40.Hw , 03.50.De , 41.20.-q 


\section{Introduction}

Maxwell's theory of classical electromagnetic phenomena employs linear partial differential equations to describe the behaviour of fields in source-free regions of the vacuum. The extension of the theory to fields in material media may involve non-linear modifications arising from the complex interactions between distributions of charge at a fundamental level. However, with the advent of high-power lasers and high-field gradients in plasmas one may be approaching regimes where the linear nature of Maxwell vacuum electrodynamics breaks down with attendant implications for electrodynamics in media. Certainly one expects vacuum polarization induced by quantum processes to intrude when electric field strengths approach $1.3 \times 10^{16} \mathrm{~V} / \mathrm{cm}$. This is still some orders of magnitude greater than current field intensities in pulsed lasers so it is of interest to enquire whether classical effects of nonlinear vacuum electrodynamics [1, [2] may yield experimental signatures before the need to accommodate quantum phenomena. The role of non-linear vacuum electrodynamics at a fundamental level may offer new insights into the problem of classical radiation reaction on particles and high-intensity field-particle interactions in plasmas.

One difficulty in assessing the significance of non-linear vacuum electrodynamics is in constructing a tractable generalisation of Maxwell's theory that is amenable on some scale to experimental verification. In this note we suggest that an approach involving the self-consistent relativistic motion of a smooth fluid-like distribution of matter (composed of a large number of charged or neutral particles) in an electromagnetic field offers a viable theoretical framework in which to explore experimental consequences. Such a framework has many applications including the motion of particles in modern accelerators and plasmas as well as phenomena in astrophysical contexts such as in the environment of magnetars, quasars and gamma-ray bursts. In the following it is assumed that the electromagnetic field is a 2 -form $F$ on space-time with a metric tensor field

$$
g=-e^{0} \otimes e^{0}+\sum_{k=1}^{3} e^{k} \otimes e^{k}
$$

where $\left\{e^{a}\right\}$ is a local co-frame with dual basis $\left\{X_{a}\right\}$ for $a=0,1,2,3$. Furthermore it is assumed that locally $F$ can be expressed in terms of the 1 -form $A$ by $F=d A$ and that electrically charged matter interacts with the field 
via a $U(1)$ covariant interaction giving rise to a regular 4-current density 3 -form $\mathcal{J}^{U 1}$. Singular sources, such as point charges contribute a singular distributional current $\mathcal{J}_{e}^{D}$. The generalized Maxwell system for the field $F$ is taken to be

$$
\begin{gathered}
d F=0 \\
d \star G=\mathcal{J}_{e}^{D}+\mathcal{J}^{U 1}
\end{gathered}
$$

where $\star$ denotes the Hodge map associated with $g$. The 2 -form $G$ is related to $F$ by a constitutive relation which for non-linear vacuum electrodynamics is non-linear. In this note it is assumed that such a relation is local and takes the form

$$
\star G=Z_{1}(F, g)
$$

for some tensor $Z_{1}$. Furthermore it is assumed that the 3 -form $\mathcal{J}^{U 1}$ may depend locally on $F, g$ and a unit time-like 4-vector field $V$ describing a smooth flow of matter on space-time:

$$
\mathcal{J}^{U 1}=Z_{2}(F, V, g)
$$

In the following the 1 -form $\widetilde{V}$ is related directly to the vector field $V$ by the metric. It is defined by the relation $\widetilde{V}(X)=i_{X} \widetilde{V}=g(V, X)$ for all vector fields $V$ and $i_{X_{a}}$ is abbreviated $i_{a}$.

In the absence of matter the constitutive tensor $Z_{1}$ can be derived from an action of the form

$$
S_{1}[A, g]=\int_{M} \hat{\mathcal{L}}(F, \nabla F, \cdots, g) \star 1
$$

involving some Lagrangian 0-form $\hat{\mathcal{L}}(F, \nabla F, \cdots, g)$. If one further restricts to

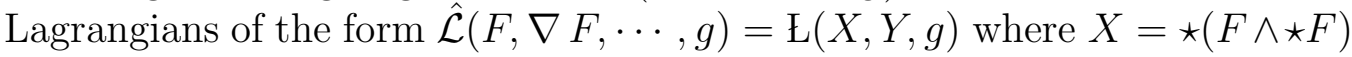
and $Y=\star(F \wedge F)$ then

$$
\star G=Z_{1}(F, g)=2 \star F \mathrm{Ł}_{X}+2 F \mathrm{Ł}_{Y}
$$

where $\mathrm{E}_{X}=\frac{\partial \mathrm{E}}{\partial X}$ and $\mathrm{E}_{Y}=\frac{\partial \mathrm{E}}{\partial Y}$. The vacuum stress-energy-momentum tensor $T^{(N L E D)}$ follows from metric variations of $S_{1}[A, g]$ as $T^{(N L E D)}=\left(\star \tau_{a}\right) \otimes e^{a}$ where

$$
\tau_{a}=M \star e_{a}+N \tau_{a}^{(L E D)}
$$


with $M=\left(\mathrm{E}-X \mathrm{七}_{X}-Y \mathrm{Ł}_{Y}\right), N=2 \mathrm{E}_{X}$ and

$$
\tau_{a}^{(L E D)}=\frac{1}{2}\left(i_{a} F \wedge \star F-i_{a} \star F \wedge F\right) .
$$

The dependence of the forms $\tau_{a}^{(L E D)}$ on $F$ is the same as that in Maxwell's linear electrodynamics in the vacuum.

\section{Charged Thermodynamic Fluids}

Consider matter with proper mass-energy density $\rho$, proper charge density $\rho_{e}$ and convective electric 4-current $\mathcal{J}^{U 1}=\rho_{e} \star \widetilde{V}$. Its equation of motion is given by

$$
\nabla \cdot T_{(t o t a l)}=0
$$

for some total stress-energy-momentum tensor

$$
T_{(\text {total })}=T^{(N L E D)}+T_{(\text {fluid })} .
$$

For a fluid without dissipation but thermodynamic pressure $p$ it will be assumed that

$$
T_{(\text {fluid })}=(\rho+p) \tilde{V} \otimes \tilde{V}+p g .
$$

It follows immediately that

$$
\nabla \widetilde{T_{(\text {fluid })}}=V \nabla \cdot((\rho+p) V)+(\rho+p) \nabla_{V} V+\widetilde{d p} .
$$

The divergence of $T^{(N L E D)}$ is more complicated and it is convenient to introduce some abbreviations. For any vector field $Q$ with ortho-normal components $Q^{a}$ write $\tau_{Q}^{(L E D)}=\tau_{a}^{(L E D)} Q^{a}$ so

$$
\nabla \cdot T^{(N L E D)}=d M+\star \tau_{\overline{d N}}^{(L E D)}-i_{\hat{J}} F
$$

where

$$
\hat{J}=\mathcal{J}^{U 1}-\star(d N \wedge \star F)-\star(d L \wedge F)
$$

with $L=2 \mathrm{E}_{Y}$. Equation (10) yields

$$
d M+\star \tau_{\widetilde{d N}}^{(L E D)}-i_{\hat{J}} F V \nabla \cdot((\rho+p) V)+(\rho+p) \nabla_{V} V+\widetilde{d p}=0
$$


which upon contracting with $V$ gives the tangential component continuity equation

$$
(p+\rho) \nabla \cdot V=i_{V} d M-i_{V} \star \tau_{\widetilde{d N}}^{(L E D)}-V(\rho)-i_{V} i_{\hat{J}} F
$$

Substituting this into (16) yields, in terms of the projection operator $\Pi_{V}=$ $\left(1+\tilde{V} i_{V}\right)$, the relativistic fluid equation of motion

$$
(\rho+p) \widetilde{\nabla_{V} V}=\Pi_{V} \mathcal{P}
$$

where the total pressure 1-form

$$
\mathcal{P}=i_{\hat{J}} F-d M-d p-\star \tau_{\widetilde{d N}}^{(L E D)} .
$$

The proper mass-energy density $\rho\left(\rho_{m}, p\right)$ can be expressed in terms of the proper mass density $\rho_{m}$ and the pressure $p$ given a specific internal energy function $\mathcal{E}\left(\rho_{m}, p\right)$ :

$$
\rho\left(\rho_{m}, p\right)=\rho_{m}\left(1+\mathcal{E}\left(\rho_{m}, p\right)\right)
$$

The thermodynamic temperature $T$ and entropy $\mathcal{S}$ of the fluid are defined via the relation

$$
T d \mathcal{S}=d \mathcal{E}+p d\left(\frac{1}{\rho_{m}}\right)
$$

which may be expressed in terms of $d \rho_{m}$ and $d p$.

\section{Born-Infeld Fluids}

Born-Infeld non-linear vacuum electrodynamics has much to recommend it [3], 4]. Aside from its historic significance it is thought to encapsulate aspects of effective string theory [5], 6] including electromagnetic duality covariance. Here it will be adopted in a gravity free environment and its salient features explored in the context of the relativistic fluid. The Lagrangian takes the form

$$
\mathrm{E}(X, Y, g)=\frac{\epsilon_{0}}{\kappa^{2}}(1-\sqrt{\Delta(X, Y)})
$$

with

$$
\begin{gathered}
\Delta(X, Y)=1-\kappa^{2} X-\frac{\kappa^{4}}{4} Y^{2} \\
2 \mathrm{E}_{X}=\frac{\epsilon_{0}}{\kappa^{2} \sqrt{\Delta}}
\end{gathered}
$$




$$
2 \mathrm{E}_{Y}=\frac{\epsilon_{0} Y}{2 \sqrt{\Delta}}
$$

and is governed by a new constant of nature 11 . It follows that the vacuum constitutive relation is

$$
\star G=\frac{\epsilon_{0}}{\kappa^{2} \sqrt{\Delta}}\left(\star F+\frac{\kappa^{2}}{2} Y F\right)
$$

and the fluid system can be rewritten as

$$
(\rho+p) \widehat{\nabla_{V} V}=\Pi_{V}\left(i_{\hat{J}} F+i_{\eta} F-d p-\xi\right)
$$

where

$$
\begin{gathered}
\xi \equiv d M+\star \tau_{\overparen{d N}}^{(L E D)}, \\
\tilde{\eta} \equiv \star(d N \wedge \star F)+\star(d L \wedge F) .
\end{gathered}
$$

With $\mathcal{J}^{U 1}=\rho_{e} V$ one has $\Pi_{V} i_{\hat{J}} F=\rho_{e} i_{V} F$ and (3) yields:

$$
(\rho+p) \widetilde{\nabla_{V} V}=\rho_{e} i_{V} F+\Pi_{V}\left(i_{\eta} F-d p-\xi\right) .
$$

In Maxwell electrodynamics

$$
\mathcal{L}=X
$$

and hence $\eta=\xi=0$ and the system reduces to:

$$
\begin{gathered}
V \cdot V=-1, \quad d F=0, \quad d \star G=\rho_{e} \star \tilde{V}, \quad G=\epsilon_{0} F, \\
(\rho+p) \nabla_{V} \tilde{V}=\rho_{e} F(V)-d p \\
(\rho+p) \nabla \cdot V=-V(\rho)
\end{gathered}
$$

exhibiting flow under the Lorentz force $\rho_{e} F(V)$ and thermodynamic pressure gradients.

By contrast, in the Born-Infeld electrodynamics, even in the absence of electrically charged matter couplings contributing to $\hat{J}$ via the $U(1)$ electric current $\mathcal{J}^{U 1}$, there is a non-zero Born-Infeld electro-dynamic pressure $\Pi_{V}(\xi-$ $\left.i_{\eta} F\right)$ contributing to the total pressure on the fluid:

$$
(\rho+p) \widetilde{\nabla_{V} V}=-\Pi_{V}\left(\xi-i_{\eta} F\right)-\Pi_{V} d p .
$$

\footnotetext{
${ }^{1}$ The fundamental constant $\kappa$ has SI dimensions $\left[\frac{Q T^{2}}{M L}\right]$ and $\epsilon_{0}$ is the permittivity of free space.
} 
This equation together with the above continuity equation:

$$
(\rho+p) \nabla \cdot V=-V(\rho)+i_{V}\left(\xi+i_{\eta} F\right)
$$

and Born-Infeld field equations in the absence of singularities

$$
d F=0 \quad, \quad d \star G=0
$$

constitute the equations for a coupled $U(1)$-neutral, relativistic Born-Infeld thermodynamic fluid [7], [8]. If one can neglect collisions and internal energy, one has a $U(1)$-neutral cold, thermodynamically inert fluid (dust) satisfying (29), (27) and (28) with $p=0, \rho=\rho_{m}$. The remaining electrodynamic pressures may arise whenever $\eta$ and $\xi$ are non-zero for fields $F$ such that $d X \neq 0$ and $d Y \neq 0$.

Such pressures can arise from solutions in Born-Infeld electrodynamics in Minkowski space-time with plane propagating waves superposed with a uniform static magnetic field in vacuo [9]. A particular case is a magnetic field transverse to the direction of propagation of a plane wave with an arbitrary smooth longitudinal profile $\mathcal{E}(z)$ :

$$
F=\mathcal{E}(z-v t) d(z-v t) \wedge d x-B d y \wedge d z
$$

describing the electric and magnetic fields in an inertial frame:

$$
\mathbf{e}=-\frac{v}{c} \mathcal{E}(z-v t) \hat{\boldsymbol{x}}, \quad \mathbf{b}=\frac{\mathcal{E}(z-v t)}{c} \hat{\boldsymbol{y}}-\frac{B}{c} \hat{\boldsymbol{x}}
$$

where

$$
v=\frac{c}{\sqrt{1+c^{2} \kappa^{2} B^{2}}} .
$$

Thus the static magnetic field with magnitude $B$ slows down the propagating electromagnetic field with amplitude proportional to $\mathcal{E}$ to a phase speed $v<c$ in vacuo. Since this retardation is cumulative it may be amenable to experimental analysis with laboratory magnetic fields. If one sets $\kappa \simeq \frac{\epsilon_{0} r_{0}^{2}}{e}$ in terms of the classical radius of the electron 2 then the Born-Infeld electron model bounds $\kappa<10^{-22}$. The wave transit time difference between when the static field is switched on and off in a magnet region of length $L_{0}$ is

$$
\tau=\frac{L_{0}}{2} \kappa|B|
$$

\footnotetext{
${ }^{2} r_{0}=\frac{e^{2}}{4 \pi \epsilon_{0} m_{e} c^{2}} \simeq 2.8 E-15 \mathrm{~m}$
} 
So for $\kappa<10^{-22}, L$ in metres and $|B|$ in Tesla

$$
\tau<\frac{L}{2}|B| 10^{-6} \mathrm{ps}
$$

where $1 \mathrm{ps}=10^{-12}$ sec. This suggests that a terrestrial experiment could be used to place bounds on the coupling $\kappa$.

\section{Conclusions}

A general model of a charged fluid interacting with an electromagnetic field whose vacuum properties are governed by a Lagrangian generalizing Maxwell's theory has been devised. It exhibits new pressure gradients of a purely electrodynamic origin in addition to those expected from Maxwell's theory. These forces may exist even when the fluid is electrically neutral in the vacuum. The particular case of a Born-Infeld fluid has been chosen to illustrate the existence of these forces when the fluid moves in a background static magnetic field on which a plane wave of arbitrary longitudinal profile propagates. The properties of this wave offer a means to bound the fundamental Born-Infeld coupling. Once one has bounds on $\kappa$ it is proposed that the framework above offers a new and intriguing avenue to explore the effects of non-linear vacuum electrodynamics in high field regimes that may become accessible to observation before the breakdown of classical electrodynamics. 


\section{Acknowledgment}

RWT is grateful to colleagues at the Cockcroft Institute for valuable discussions, to the EPSRC for a Springboard Fellowship for financial support

for this research which is part of the Alpha-X collaboration. We thank Koç University for its hospitality where part of this research is carried out and the Turkish Academy of Sciences (TUBA) for a travel grant.

\section{References}

[1] M. Born, L. Infeld, Proc. Roy. Soc. A144 (1934) 425

[2] J. Plebankski, Lectures on Non-linear Electrodynamics (NORDITA, Copenhagen, 1968)

[3] G. Boillat, J. Math. Phys. 11 (1970) 941

[4] S. Deser, J. McCarthy, Ö. Sarığlu, Class. Q. Grav. 16 (1999) 841

[5] E. Fradkin, A. A. Tseytlin, Phys. Lett. 163B (1985) 123

[6] G. W. Gibbons, C. A. R. Herderio, Phys. Rev. D63 (2001) 064006

[7] P. A. M. Dirac, Proc. Roy. Soc. A257 (1960) 32

[8] D. Chruściński, Phys. Lett. A240(1998) 8

[9] M. Aiello, G. R. Bengochea, R. Ferraro, Phys. Lett. A361(2007) 9 